\title{
Swift/XRT observations of unidentified INTEGRAL/IBIS sources
}

\section{R. Landi*}

INAF/IASF Bologna, Via P. Gobetti 101, 40129 Bologna, Italy

E-mail: Landiliasfbo.inaf.it

\section{Bassani}

INAF/IASF Bologna, Via P. Gobetti 101, 40129 Bologna, Italy

E-mail: bassanidiasfbo.inaf.it

\section{A. Malizia}

INAF/IASF Bologna, Via P. Gobetti 101, 40129 Bologna, Italy

E-mail: maliziadiasfbo.inaf.it

\section{A. Bazzano}

INAF/IASF Roma, Via Fosso del Cavaliere 100, 00133 Roma, Italy

E-mail: angela.bazzanodiasf-roma.inaf.it

\section{Fiocchi}

INAF/IASF Roma, Via Fosso del Cavaliere 100, 00133 Roma, Italy

E-mail: mariateresa.fiocchidiasf-roma.inaf.it

\section{A. J. Bird}

School of Physics and Astronomy, University of Southampton, Highfield, SO17 1BJ, UK

E-mail: A.J.Birdesoton.ac.uk

The most recent INTEGRAL/IBIS surveys list many unidentified sources, i.e. those lacking an optical and X-ray counterpart. The X-ray telescope (XRT, 0.3-10 keV) on board Swift, thanks to its few arcseconds source location accuracy, is a powerful tool with which the X-ray counterparts to these IBIS sources can be searched for and studied. In fact, the refinement of the source position is of key importance to perform more efficient and reliable follow-up observations at other wavelengths (optical, UV, radio). In this work, we present the results of the XRT observations for 6 new gamma-ray sources (IGR J12134-6015, IGR J14043-6148, IGR J14488-4008, IGR J16058-7253, IGRJ17488-2338, and SWIFT J2037.2+4151), showing how the combination, when possible, of the XRT and IBIS data gives us some hints about the nature of these objects. For all sources, but one (IGR J16058-7253), we find a likely soft counterpart, but optical follow-up observations are needed to classify these objects. We propose a Galactic nature for IGR J12134-6015 and SWIFT J2037.2+4151, while IGR J14488-4008, IGR J16058-7253, and IGRJ17488-2338 seem to have properties typical of type 2 AGN. For IGR J14043-6148, the information available so far prevents us from drawing any secure conclusion about its nature.

The Extreme and Variable High Energy Sky - extremesky2011,

September 19-23, 2011

Chia Laguna (Cagliari) Italy

\footnotetext{
* Speaker.
} 
Table 1: XRT position of the likely counterpart to the IBIS sources and its proposed nature.

\begin{tabular}{|c|c|c|c|c|}
\hline Source & $\begin{array}{l}\text { R.A. } \\
\text { (J2000) }\end{array}$ & $\begin{array}{c}\text { Dec. } \\
(\mathrm{J} 2000)\end{array}$ & $\begin{array}{c}\text { Error } \\
(\operatorname{arcsec})\end{array}$ & Type \\
\hline IGR J12134-6015 & $12^{\mathrm{h}} 13^{\mathrm{m}} 23^{\mathrm{s}} .95$ & $-60^{\circ} 15^{\prime} 15^{\prime \prime} .5$ & 3.6 & $\begin{array}{l}\text { Galactic object } \\
\text { (HMXB ?) }\end{array}$ \\
\hline IGR J14043-6148 & $14^{\mathrm{h}} 04^{\mathrm{m}} 29^{\mathrm{s}} .63$ & $-61^{\circ} 47^{\prime} 19^{\prime \prime} .7$ & 4.5 & AGN or SNR ? \\
\hline IGR J14488-4008 & $14^{\mathrm{h}} 48^{\mathrm{m}} 50^{\mathrm{s}} .98$ & $-40^{\circ} 08^{\prime} 47^{\prime \prime} .1$ & 3.8 & AGN? \\
\hline \multirow[t]{2}{*}{ IGR J16058-7253 } & $16^{\mathrm{h}} 06^{\mathrm{m}} 06^{\mathrm{s}} .66$ & $-72^{\circ} 52^{\prime} 42^{\prime \prime} .3$ & 4.1 & likely type 2 AGN \\
\hline & $16^{\mathrm{h}} 05^{\mathrm{m}} 22^{\mathrm{s}} .84$ & $-72^{\circ} 53^{\prime} 55^{\prime \prime} .4$ & 3.8 & type 2 AGN \\
\hline IGR J17488-2338 & $17^{\mathrm{h}} 48^{\mathrm{m}} 38^{\mathrm{s}} .84$ & $-23^{\circ} 35^{\prime} 20^{\prime \prime} .8$ & 4.5 & type 2 AGN? \\
\hline SWIFT J2037.2+4151 & $20^{\mathrm{h}} 37^{\mathrm{m}} 05^{\mathrm{s}} .51$ & $+41^{\circ} 50^{\prime} 05^{\prime \prime} .7$ & 3.5 & $\begin{array}{l}\text { X-ray binary } \\
\text { (CV or HMXB ?) }\end{array}$ \\
\hline
\end{tabular}
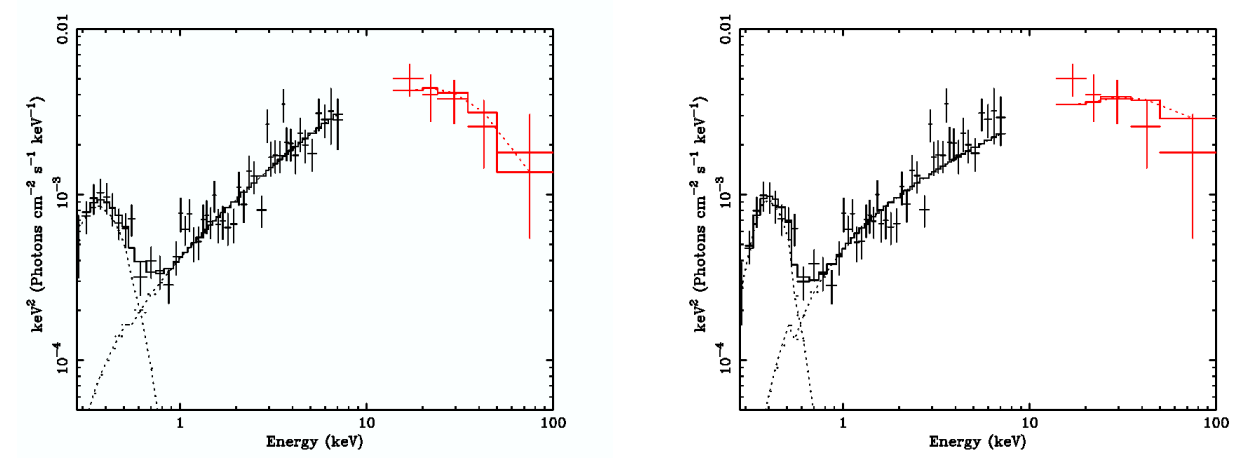

Figure 1: Broad-band spectrum of IGR J12134-6015 fitted with model A (left panel), and model B (right panel), see text.

\section{IGR J12134-6015}

XRT reveals only one X-ray source (see Table 1) within the IBIS and BAT positional uncertainties (Krivonos et al. 2010; Baumgartner et al. 2010). This source has a counterpart listed in the 2MASS (2 Micron All Sky Survey, Skrutskie et al. 2006), 2MASS J12132397-6015169, having magnitudes $J \sim 16.4, H \sim 16.5$, and $K \sim 15.5$. Within the XRT position we also find a ROSAT Faint source 1RXS J121324.5-601458 and the XMM Slew source XMMSL1 J121323.5-601517. The broad-band spectrum, obtained by combining the average XRT spectrum and the BAT data, is well described both with a model (model A, see Figure 1, left panel) including intrinsic absorption $\left(N_{\mathrm{H}}=\left(3.8_{-3.7}^{+5.4}\right) \times 10^{20} \mathrm{~cm}^{-2}\right)$, blackbody emission $\left(k T_{\mathrm{bb}}=62_{-12}^{+9} \mathrm{keV}\right)$, and a cut-off power law $\left(\Gamma=0.85_{-0.09}^{+0.17}, E_{\mathrm{C}}=18_{-7}^{+13} \mathrm{keV}\right)$ and a model (model B, see Figure 1, right panel) consisting of an intrinsic absorption $\left(N_{\mathrm{H}}=(1.1 \pm 0.5) \times 10^{21} \mathrm{~cm}^{-2}\right)$, a blackbody $\left(k T_{\mathrm{bb}}=48_{-7}^{+9} \mathrm{keV}\right)$ plus thermal bremsstrahlung component $\left(k T_{\text {brems }}=46_{-20}^{+76} \mathrm{keV}\right)$.

The analysis of each XRT observations indicates strong variability (by a factor of 3 ) on a timescale of a few days. This source behaviour combined with the results of the broad-band spectral analysis leads us to suggest a likely Galactic nature for this objects, perhaps a High Mass X-ray Binary (HMXB). 

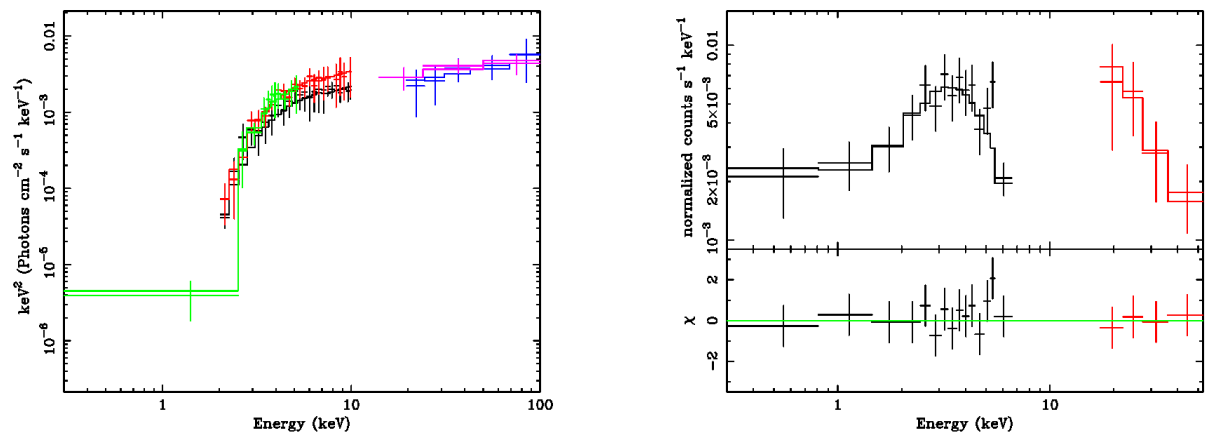

Figure 2: Broad-band spectrum of IGR J14043-6148 fitted with an absorbed power law (left panel), and of IGR J14488-4008 modelled with an absorbed power law plus a second power law passing through Galactic absorption to account for the excess observed below $2 \mathrm{keV}$ (right panel).

\section{IGR J14043-6148}

This source has been detected by IBIS (Bird et al. 2010), BAT (Baumgartner et al. 2010) and by BeppoSAX during a scan of the Galactic plane. There is only one X-ray source (see Table 1) whose position is compatible both with IBIS and BAT error circles. Within the XRT positional uncertainty we find an object belonging to the United States Naval Observatory (USNO-B1.0, Monet et al. 2003) catalogue, USNO-B1.0 0282-0474947, with magnitude $R \sim 14.9-16.5$, which is also reported in the 2MASS (2MASS J14042968-6147201, $J \sim 16.2, H \sim 13.2$, and $K \sim 11.2$ ) and GLIMPSE (Galactic Legacy Infrared Midplane Survey Extraordinaire, Benjamin et al. 2003), G311.4524-00.1324, with magnitude at $8 \mu \sim 8.1$. The XRT location is also compatible with a XMM-Newton serendipitous source (2XMM J140429.4-614727) and with a non thermal radio source (G311.45-0.13) having a $6 \mathrm{~cm}$ flux of 0.5 Jy (Caswell \& Barnes 1985); this object is slightly resolved and has been proposed to be either a small SNR or a background galaxy.

The broad-band spectrum, obtained by combing the XRT, MECS, IBIS and BAT data together, is shown in Figure 2 (left panel). The data are well modelled with an absorbed $\left(N_{\mathrm{H}}=7.6_{-2.4}^{+3.1} \times 10^{22}\right.$ $\mathrm{cm}^{-2}$ ) power law with a photon index $\Gamma=1.63 \pm 0.33$. No significant variability is found for this object being the cross-calibration constants compatible with unity. The observed average 2-10 $\mathrm{keV}$ and 20-100 keV fluxes are $\sim 3 \times 10^{-12}$ and $\sim 1 \times 10^{-11} \mathrm{erg} \mathrm{cm}^{-2} \mathrm{~s}^{-1}$, respectively.

Given the X-ray characteristics, we favour the AGN nature for the object, although we cannot exclude that it could be a composite SNR/pulsar wind nebulae.

\section{IGR J14488-4008}

The X-ray position of the only source still visible above $3 \mathrm{keV}$ (see Table 1), within the IBIS and BAT error circles (Bird et al. 2010; Cusumano et al. 2010), is consistent with the radio source CRATES J1448-4008 (NED03) having a $6 \mathrm{~cm}$ flux of $126 \mathrm{mJy}$ and with NVSS J144851400846 having a $20 \mathrm{~cm}$ flux of $48.0 \pm 1.9 \mathrm{mJy}$. In the optical band this source coincides with the USNO-A2.0 object (USNO-A2.0 0450_18513818) having $R \sim 14.0$ and $B \sim 14.7$, while in infrared is associated with a 2MASS source (2MASS J14485097-4008456) displaying $J \sim 16.7$, 

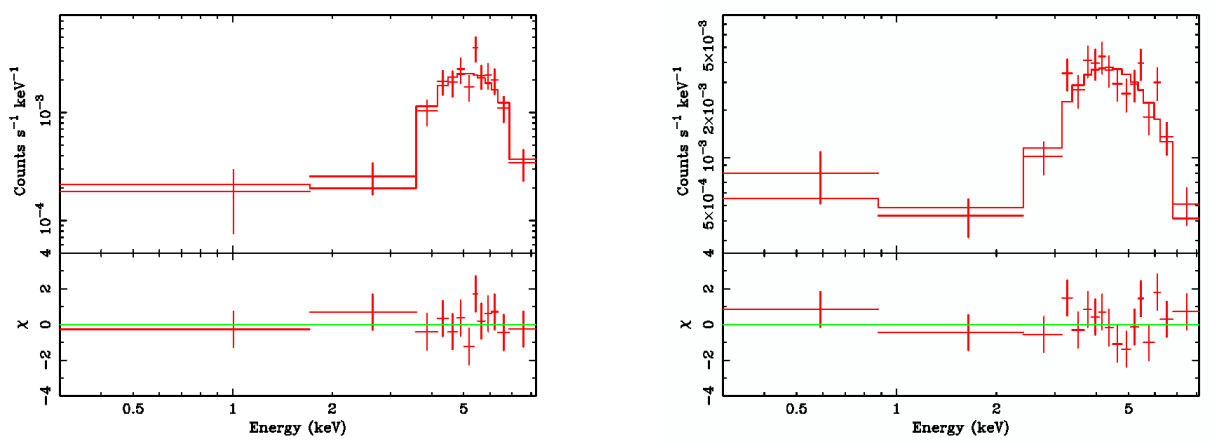

Figure 3: XRT spectra of the two sources detected within the IBIS error circle of IGR J16058-7253: both source \#1 (left panel) and source \#2 (right panel) data are modelled with an absorbed power law.

$H \sim 12.7$, and $K \sim 11.6$. The XRT and IBIS data are well fitted (see Figure 2 , right panel) using a double power law model with indices tied together and with a value $\Gamma=2.32_{-0.29}^{+0.43}$; in addition to the Galactic absorption, data requires an intrinsic column density $N_{\mathrm{H}}=\left(6.4_{-1.6}^{+1.9}\right) \times 10^{22} \mathrm{~cm}^{-2}$. The observed 2-10 and 20-100 keV fluxes are $\sim 2.5 \times 10^{-12}$ and $\sim 6.2 \times 10^{-12} \mathrm{erg} \mathrm{cm}^{-2} \mathrm{~s}^{-1}$, respectively.

Based on the multiwaveband characteristics, we propose for IGR J14488-4008 a type 2 AGN classification.

\section{IGR J16058-7253}

This source has been detected by IBIS (Krivonos et al. 2010) and BAT (Baumgartner et al. 2010). There are two sources detected by XRT and located within the IBIS and BAT positional uncertainties (see Table 1). The XRT position of source \#1 is compatible with that of a USNO-A2.0 object (USNO-A2.0 0150_16011427) with magnitudes $R \sim 15.7$ and $B \sim 16.8$ and a 2MASS extended source (2MASX J16060682-7252418, with magnitudes $J \sim 15.0, H \sim 14.2$, and $K \sim 13.1$ ), classified as a galaxy in NED. Source \#2 again coincides with a 2 MASS extended source (2MASX J16052330-7253565, with magnitudes $J \sim 13.3, H \sim 12.4$, and $K \sim 12.2$ ) also classified as a galaxy in NED, and associated with a USNO-A2.0 object (USNO-A2.0 0150_15991912) with magnitudes $R \sim 11.9$ and $B \sim 12.0$. This source is also reported as a radio emitter in the Sydney University Molonglo Sky Survey (SUMSS, Mauch et al. 2003) catalogue with a flux of $9.2 \pm 0.9 \mathrm{mJy}$ at 36 $\mathrm{cm}$.

Both objects are well fitted with an absorbed power law (see Figure 3): $N_{\mathrm{H}}=\left(3.8_{-1.6}^{+1.1}\right) \times 10^{23}$ $\mathrm{cm}^{-2}$ and $\Gamma=1.75_{-0.29}^{+0.35}$ for source $\# 1$, and $N_{\mathrm{H}}=\left(1.7_{-0.5}^{+0.6}\right) \times 10^{23} \mathrm{~cm}^{-2}$ and $\Gamma=1.90_{-0.49}^{+0.35}$ for source \#2. Also the observed 2-10 keV fluxes of the two source are comparable: $\sim 2.9$ and $\sim 3.3 \times 10^{-12}$ erg cm $\mathrm{cm}^{-2} \mathrm{~s}^{-1}$ for source \#1 and \#2, respectively. Interestingly, the extrapolation of the $20-100 \mathrm{keV}$ flux from the XRT data set reproduces the total IBIS observed flux $\left(\sim 1.7 \times 10^{-11} \mathrm{erg} \mathrm{cm}^{-2} \mathrm{~s}^{-1}\right)$, suggesting that both objects might contribute to the high energy emission. Preliminary result from optical follow-up observations (Masetti \& Parisi, private communication) indicate source \#2 as a type 2 AGN. The similarity of the spectra of the two sources leads us to favour a likely type 2 AGN nature also for source \#1. 

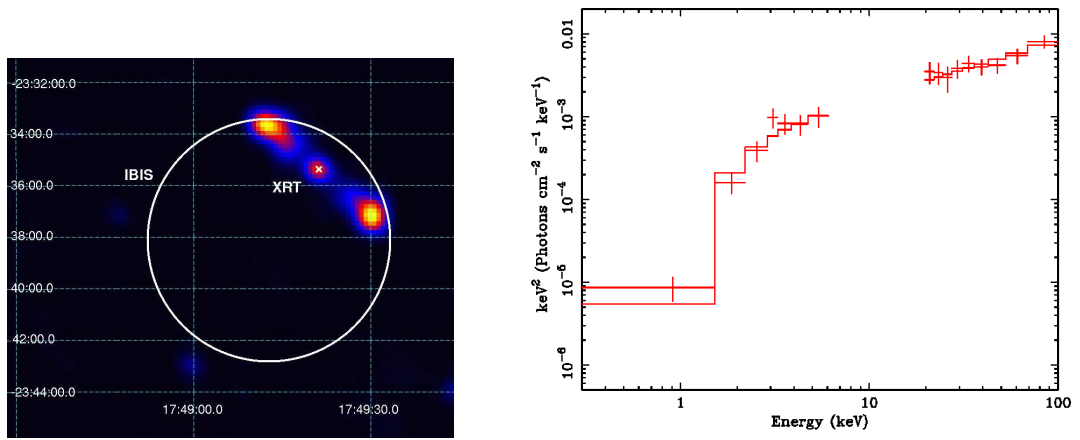

Figure 4: NVSS radio map of the region surrounding IGR J17488-2338 (Stephen et al. 2010). The white circle represents the $90 \%$ IBIS error circle, while the white cross shows the positions of the XRT detection (left panel); broad-band spectrum modelled with an absorbed power law (right panel).

\section{IGR J17488-2338}

There is only one X-ray source detected by XRT and located within the IBIS positional uncertainty (see Table 1). The XRT position of source \#1 is compatible with that of two USNO-A2.0 objects: USNO-A2.0 0600_28864869 (with magnitudes $R \sim 17.5$ and $B \sim 19.1$ ) and USNO-A2.0 0600_28865103 (with magnitudes $R \sim 17.2$ and $B \sim 19.1$ ). The $X$-ray source also coincides with a XMM-Newton Slew object (XMMSL1 J174838.8-233527) and has a radio counterpart (see Figure 4 left panel) belonging to the NVSS catalogue (NVSS J174839-233517) with a $20 \mathrm{~cm}$ flux of $131.4 \pm 4.8 \mathrm{mJy}$.

The broad-band spectrum (average XRT spectrum combined to the IBIS one during outburst) is well described (see Figure $\sharp$, right panel) by an absorbed $\left(N_{\mathrm{H}}=\left(1.3_{-0.8}^{+1.3}\right) \times 10^{22} \mathrm{~cm}^{-2}\right)$ having a photon index $\Gamma=1.30_{-0.32}^{+0.31}$ and observed $2-10$ and $20-100 \mathrm{keV}$ fluxes of $\sim 2.3 \times 10^{-12}$ and $\sim 1.4 \times 10^{-11} \mathrm{erg} \mathrm{cm}^{-2} \mathrm{~s}^{-1}$, respectively. Despite being classified as a variable object in the fourth catalogue (Bird et a. 2010), the cross-calibration constant turned out to be compatible with unity.

The spectral properties, as well as the radio double lobe morphology, suggest an AGN nature also for IGR J17488-2338, likely of type 2 .

\section{SWIFT J2037.2+4151}

This source, reported as a JEM-X object by Westergaard et al. (2006), is listed in the IBIS survey (Krivonos et al. 2010) and in the Swift/BAT 22 month catalogue (Tueller et al. 2010), in which is reported as a transient object. Also in this case inside the IBIS and JEM-X error circles, XRT detects only one X-ray object (see Table 1). Within the XRT positional uncertainty we find a 2MASS source (2MASS 20370560+4150051) detected with magnitudes $J \sim 16.2, H \sim 13.5$, and $K \sim 12.1$. No optical counterpart has been found for SWIFT J2037.2+4151.

A good fit to the average $\mathrm{X}$-ray spectrum is provided by an absorbed $\left(N_{\mathrm{H}}=(3.2 \pm 0.4) \times 10^{22}\right.$ $\left.\mathrm{cm}^{-2}\right)$ power law $\left(\Gamma=0.74_{-0.13}^{+0.16}\right)$ plus an iron line at $E_{\text {Line }}=6.7 \pm 0.1 \mathrm{keV}$ with an $E W=575_{-141}^{+161}$ $\mathrm{eV}$. Comparison between XRT observations indicates variability in the source flux by a factor of $\sim 1.5$ over a period around one. 

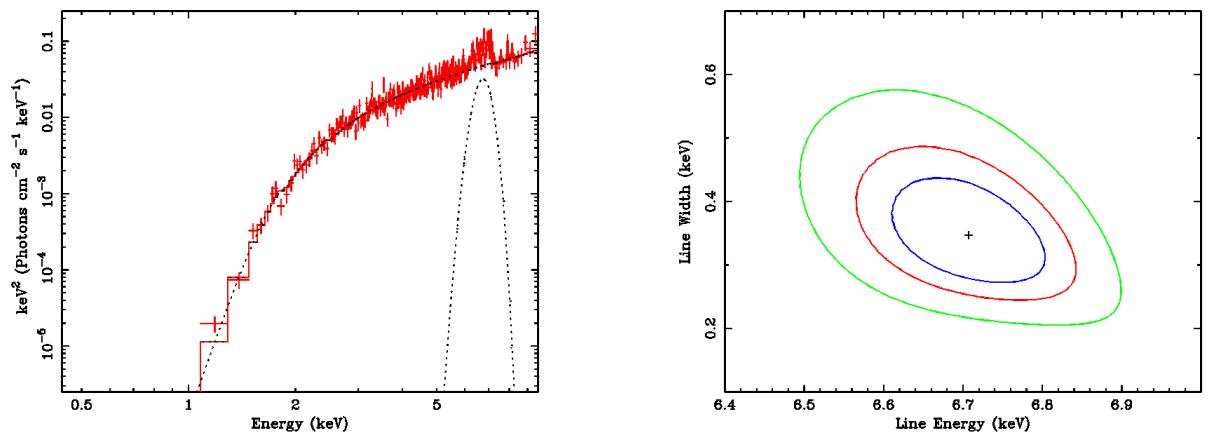

Figure 5: XRT spectrum of SWIFT J2037.2+4151 fitted with an absorbed power law plus an iron line (left panel); countours plot showing the line energy versus the line width (right panel).

Based on these spectral properties we favour a Galactic nature for SWIFT J2037.2+4151: it is probably an X-ray binary, either a cataclysmic variables or a HMXB.

\section{Conclusions}

In this work, we show how follow-up observations in X-rays are of key importance to search for counterparts of high energy emitters. The cross-correlation between the IBIS surveys and the Swift/XRT data archive allowed us to pinpoint unambiguously the soft X-ray counterpart of six IBIS sources. Two objects (IGR J12134-6015 and SWIFT J2037.2+4151) seem to have a Galactic nature, three objects (IGR J14488-4008, IGR J16058-7253 and IGRJ17488-2338) exhibit properties typical of AGN. The remaining source, IGR J14043-6148, needs to be further studied in order to assess its nature; at this stage, although we favour an AGN nature, a composite SNR/pulsar wind nebulae cannot be excluded.

\section{References}

[1] Baumgartner, W. H., et al., 2010, ApJS submitted

[2] Benjamin R. A., Churchwell E., Babler B. L., et al., 2003, PASP, 115, 953

[3] Bird A. J., A. Bazzano, L. Bassani, et al., 2010, ApJS, 186, 1

[4] Caswell J. L. \& Barnes P. J., 1985, MNRAS, 216, 753)

[5] Cusumano, G., La Parola, V., Segreto, A., et al., 2010, A\&A, 510, A48

[6] Krivonos, R., Tsygankov, S., Revnivtsev, M., et al., 2010, A\&A, 523, 61

[7] Mauch, T., Murphy, T., Buttery, H.J., et al., 2003, MNRAS, 342, 1117

[8] Monet, D. G., Levine, S. E., Canzian, B., Ables, et al. 2003, AJ, 125, 984

[9] Skrutskie, M. F., Cutri, R. M., Stiening, R., et al., 2006, AJ, 131, 1163

[10] Stephen J. B., Landi, R., Masetti, N., et al. 2010, ATel \#2441

[11] Tueller, J., Baumgartner, W. H., Markwardt, C. B., et al., 2010, ApJS, 186, 378

[12] Westergaard, N. J., Budtz-Jorgensen, C., Chenevez, J., et al. 2006, ATel \#967 